\title{
FRAMEWORKS SYMMETRY AND RIGIDITY
}

\author{
J. C. OWEN AND S. C. POWER
}

\begin{abstract}
Symmetry equations are obtained for the rigidity matrix of a bar-joint framework in $\mathbb{R}^{d}$. These form the basis for a short proof of the Fowler-Guest symmetry group generalisation of the Calladine-Maxwell counting rules. Similar symmetry equations are obtained for the Jacobian of diverse framework systems, including constrained point-line systems that appear in CAD, body-pin frameworks, hybrid systems of distance constrained objects and infinite bar-joint frameworks. This leads to generalised forms of the Fowler-Guest character formula together with counting rules in terms of counts of symmetry-fixed elements. Necessary conditions for isostaticity are obtained for asymmetric frameworks, both when symmetries are present in subframeworks and when symmetries occur in partition-derived frameworks.

Bar-joint framework; symmetry; rigidity.
\end{abstract}

\section{INTRODUCTION}

Let $(G, p)$ be a framework in $\mathbb{R}^{d}$ which, by definition, consists of an abstract graph $G=(V, E)$ and a vector $p=\left(p_{1}, \ldots, p_{v}\right)$ composed of framework points in $\mathbb{R}^{d}$. When $(G, p)$ is viewed in the natural way as a pin-jointed bar framework in $\mathbb{R}^{d}$ then there is a counting condition for bars and joints that the framework must satisfy if it is known to be isostatic, which is to say that the structure is rigid in a natural sense (infinitesimally rigid) and at the same time is not overconstrained. More generally, in the nonisostatic case, there is a single condition relating the four quantities, $v=|V|, e=|E|$, the number $m$ of nontrivial independent infinitesimal motions (also known as mechanisms), and the number $s$ of independent stresses that the structure can carry. For $d=2$ this is the extended Maxwell rule (Calladine[1])

$$
m-s=2 v-e-3
$$

while for $d=3$ one has $m-s=3 v-e-6$. The equations arise from a consideration of the kernel and cokernel of the rigidity matrix for the framework and their respective dimensions, $m$ and $s$.

Recently, in the context of the analysis of loads and stresses in symmetric structures, Fowler and Guest [2] have obtained an extended counting rule for symmetric frameworks in two and three dimensions

\footnotetext{
${ }^{1}$ Supported by a London Mathematical Society Scheme 7 Grant.
} 
and these formulae are a source of additional necessary counting conditions. In three dimensions the formula takes the elegant form

$$
\Gamma(m)-\Gamma(s)=\Gamma(v) \times \Gamma_{x y z}-\Gamma(e)-\Gamma_{x y z}-\Gamma_{R_{x} R_{y} R_{z}}
$$

where each $\Gamma$ denotes a character list for a representation of the rigid motion symmetry group $\mathcal{G}$ of the framework. Thus the equation represents a set of equations, one for each element of $\mathcal{G}$. The list $\Gamma(e)$, for example, arises from an elementary permutation representation $\rho_{e}$ of $\mathcal{G}$ on a real vector space with basis indexed by the edges of $G$. Specifically

$$
\Gamma(e)=\operatorname{trace}\left(\rho_{e}\left(g_{1}\right), \ldots, \operatorname{trace}\left(\rho_{e}\left(g_{r}\right)\right)\right.
$$

for some choice of elements $g_{1}, \ldots, g_{r}$ of $\mathcal{G}$, typically a set of generating elements with $g_{1}$ the identity element.

The significance of the formulae lie in the fact that the right-hand side is readily computable depending only on the abstract graph $G$ of the framework rather than the metrical detail. In particular trace $\left(\rho_{e}\left(g_{k}\right)\right)$ is the number of edges that are left unmoved by the symmetry $g_{k}$. The left hand side however carries information on the possibilities for stresses and flexes. Evaluating the formula for the identity element $g_{1}$ of $\mathcal{G}$ gives the Calladine-Maxwell rules. See also Ceulemans and Fowler [3] for an analogous symmetry variant of Euler's formula for polyhedra.

Our first purpose is to obtain an explicit symmetry equation

$$
R=\rho_{e}\left(g^{-1}\right) R \hat{\rho}_{v}(g), \quad g \in \mathcal{G},
$$

for the rigidity matrix $R=R(G, p)$ of a bar-joint framework in $\mathbb{R}^{d}$, which shows how the matrix intertwines representations of $\mathcal{G}$ associated with the edges and with the vertices. Here $\hat{\rho}_{v}$ is the representation $\rho_{n} \otimes \rho_{s p}$ where $\rho_{n}$ is the natural permutation representation associated with the vertices (nodes) and $\rho_{s p}$ is the usual orthogonal representation of $\mathcal{G}$ in $\mathbb{R}^{d}$. From this we obtain a simple proof of a general FowlerGuest formula for frameworks in $\mathbb{R}^{d}$. The proof is coordinate free and in fact the unitary equivalence of subrepresentations that underlies the formula may be implemented by the partially isometric part of the polar decomposition $R=U\left(R^{*} R\right)^{1 / 2}$.

Our second purpose is to show that the method is versatile and readily applicable to higher order frameworks. For example, we consider body-bar frameworks and constraint systems for geometric objects, such as the constraints of geometries arising in CAD. Once again we obtain symmetry equations, equivalent representations, character formulae and counting conditions.

Figure 1 shows a practical application in CAD for the symmetry adapted Maxwell counting rule. The geometric figure on the right shows a triangle which has reflection symmetry about a vertical axis. The graph on the right shows the corresponding constraint graph taken 

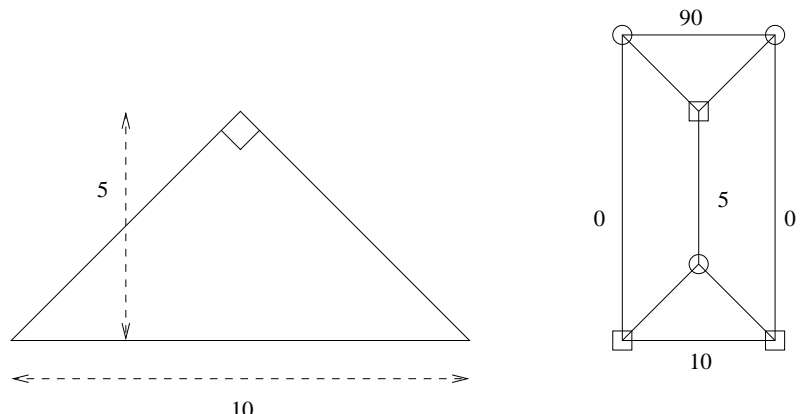

FiguRE 1. The geometric drawing has an abstract graph in which lines are represented by circular vertices and the points by square vertices. The labeled edges represent angular dimensions of 90 degrees and distances of 0,5 and 10. A count for the reflection symmetry of the graph implies the singularity of the equation system for the drawing.

from a CAD constraint solving application. In this graph the square nodes represent points, the circular nodes represent lines and the edges represent either a distance or angle dimension with the specified value or a point-line coincidence. Notice that this graph has a corresponding two-fold symmetry. The equations represented by the geometric figure and the constraint graph are singular for the following reason. If the dimension with value 5 which specifies the height of the triangle is removed then the apex of the triangle can be placed anywhere on a circle with the base of the triangle as diameter (due to the perpendicular constraint shown) and so this circle has radius 5 . Thus in the symmetric configuration shown the height dimension has attained its maximum possible value and cannot be increased. This indicates singularity in the equations. In this example $v=6$ and $e=9$ so the right hand side of Equation (1.1) evaluates to zero and is compatible with $m=s=0$. For this reflection symmetry we will show that the symmetry adapted equation takes the form $g(m)-g(s)=g(e)-1$. Since $g(e)$, the number of edges of the graph which are unchanged by the two-fold symmetry, is three, this equation requires that $m>0$ which says that the equations have at least one infinitesimal motion which means here that they are singular.

We also show how one may obtain symmetry equations and the character formula for infinite frameworks. In particular, in the case of periodic frameworks we obtain the periodic form

$$
\Gamma_{p}(m)-\Gamma_{p}(s)=\Gamma_{p}(v) \cdot \Gamma(s p)-\Gamma_{p}(e)-\Gamma_{p}(r i g),
$$

in which the trace lists are associated with finite-dimensional representations of a translation subgroup quotient of the spatial symmetry group. 
Finally we indicate how the symmetry analysis may be exploited further in two distinct ways, and even for asymmetric frameworks. In the first we consider symmetries in vertex induced subframeworks while in the second we consider latent symmetries in partition-derived frameworks. For the symmetry group identity element the properties of sub-frameworks and derived frameworks both give the same wellknown necessary requirement for non-singularity of the relevant Jacobian (such as, in two dimensions, $2 v-e \geq 3$ for every sub-graph with $e$ edges and $v$ vertices). However, symmetry in subframeworks or partition derived frameworks both give new and useful predictions.

For example we obtain in Theorem 5.1 a "singularity predictor", in the form of a set of necessary counting conditions for an isostatic framework $(G, p)$ in $\mathbb{R}^{d}$. As a simple corollary of this we observe that for a planar isostatic framework with a reflection symmetry $g$ in a subframework $X$ we have the necessary condition

$$
\left|-e_{X}^{g}+1\right| \leq 2 v_{X}-e_{X}-3 .
$$

where $e_{X}^{g}$ is the number of edges of $X$ left unmoved by $g$.

Many authors have considered group representations in the analysis of symmetric structures, often adopting symmetry adapted coordinate spaces for stresses and flexes. See, for example, Kangwai and Guest[4] and the survey Kangwai, Guest and Pellegrino[5]. In this vein irreducible group considerations were introduced in the detailed engineering calculations of Kangwai and Guest[4] and subsequently put into the useful character equational form by Fowler and Guest[2]. In contrast to this we bring out the symmetry equations as the essential feature of symmetric bar-joint frameworks and we use them to identify invariant subspaces and thereby obtain a short derivation of the formula. We note that Schulze[6] has given another rigourous proof of the FowlerGuest formula which uses a more expansive analysis of subspaces for the block diagonalisation of the rigidity matrix. Moreover interesting applications are given to noninjective frameworks with coincident vertices which are not considered here.

The usefulness of the Fowler-Guest formula has been shown in Connelly, Fowler, Guest, Schulze and Whiteley[7] where it was used to derive a complete list of the necessary counting conditions for bar-joint frameworks in two and three dimensions. These conditions are in terms of counts for the number of vertices or edges that are left unmoved by various symmetries. In our Corollary 3.2 we recover some of these results while Theorems 4.1, 4.5, 5.1 and 5.2 lead to analogous counting constraints.

For a planar isostatic framework one has $m=s=0$ and hence the necessary equality $2 v-e-3=0$. This is not a sufficient condition as one also needs subframeworks not to be overconstrained. However, it is a fundamental and celebrated theorem of Laman[8] that the necessary 
count condition $2 v-e=3$ together with the inequality $2 v_{X}-e_{X} \geq 3$ for all subgraphs $X$ is a sufficient condition for a generic framework to be isostatic. Thus necessary and sufficient conditions are known for the two dimensional generic case. We do not consider sufficiency conditions below but we note that Schulze[9] has recently obtained Laman theorems for frameworks in the plane with various symmetry.

For further background on rigidity and diverse constraint problems see, for example, Asimow and Roth[10], Connelly et al[7], Graver, Servatius and Servatius[11], Jackson and Jordan[13], Owen[14], Owen and Power[15], and Whiteley[16].

We would like to thank Simon Guest and Nadia Mazza for interesting discussions and the anonymous referees for helpful comments.

\section{Frameworks and Symmetries.}

We begin with a formal introduction to mathematical bar-joint frameworks $(G, p)$ in $\mathbb{R}^{d}$, to the rigidity matrix $R(G, p)$ and to the spatial symmetry group $\mathcal{G}$ of a framework. Also, viewing $\mathcal{G}$ as an abstract group we consider elementary representations of $\mathcal{G}$ as permutation transformations of vector spaces associated with the vertices and with the edges.

2.1. The rigidity matrix. Let $G=(V, E), n=|V|, m=|E|$ be a finite connected graph, with no multiple edges. A framework in $\mathbb{R}^{2}$ is a pair $(G, p)$ where $p=\left(p_{1}, \ldots, p_{n}\right)$ is a framework vector with framework points $p_{i}=\left(x_{i}, y_{i}\right)$ in $\mathbb{R}^{2}$ that are associated with an ordering $v_{1}, \ldots, v_{n}$ of the vertices. Thus we allow framework points to coincide. The rigidity matrix $R=R(G, p)$ for the framework $(G, p)$ is an $m \times 2 n$ real matrix whose columns are labeled by $x_{1}, y_{1}, x_{2}, y_{2}, \ldots, x_{n}, y_{n}$, and whose rows are labeled by some ordering $e_{1}, \ldots, e_{m}$ of the edges. If $e=\left(v_{i}, v_{j}\right)$ is an edge of $G$ then the matrix entries of $R$ in the row for $e$ are zero except possibly in the columns for $x_{i}, y_{i}, x_{j}, y_{j}$ where we have, respectively, $x_{i}-x_{j}, y_{i}-y_{j}, x_{j}-x_{i}, y_{j}-y_{i}, 1 \leq i \leq n$. Thus for notational economy we allow framework point coordinates to agree with their labels.

The rigidity matrix gives a linear transformation from the $2 n$-dimensional real vector space

$$
\mathcal{H}_{v}=\sum_{k=1}^{n} \oplus\left(\mathbb{R}_{x_{k}} \oplus \mathbb{R}_{y_{k}}\right),
$$

associated with the vertices, to the $m$-dimensional real vector space,

$$
\mathcal{H}_{e}=\sum_{k=1}^{m} \oplus \mathbb{R}_{e_{k}}
$$

associated with edges. Here each vector space summand $\mathbb{R}_{x_{k}}, \mathbb{R}_{y_{k}}, \mathbb{R}_{e_{k}}$ is a copy of $\mathbb{R}$. Let $\xi_{x_{k}}, \xi_{y_{k}}, 1 \leq k \leq n$, denote the standard basis for $\mathcal{H}_{v}$ and write $\xi_{e_{k}}, 1 \leq k \leq m$, for the standard basis for $\mathcal{H}_{e}$. Then the 
matrix entry $x_{i}-x_{j}$ in row $e=\left(v_{i}, v_{j}\right)$ and column $x_{i}$ is given by the standard inner product $\left\langle R \xi_{x_{i}}, \xi_{e}\right\rangle$.

The rigidity matrix $R(G, p)$ of a framework $(G, p)$ in $\mathbb{R}^{d}$ is defined in exactly the same manner. Alternatively it may be defined as one half of the Jacobian derivative of the nonlinear map from $\mathcal{H}_{v}$ to $\mathcal{H}_{e}$ which is determined by the quadratic distance equations for the framework. We adopt this viewpoint in Section 4.

The rigidity matrix derives its name from the fact that vectors $u=$ $\left(u_{i}\right)=\left(u_{x_{i}}, u_{y_{i}}\right)$ in its kernel (nullspace) are infinitesimal flexes in the following sense. They indicate directions (or velocity directions) in which for each edge the disturbances of edge length

$$
\left|p_{i}-p_{j}\right|-\mid\left(p_{i}+t u_{x_{i}}-\left(p_{j}+t u_{y_{i}}\right) \mid\right.
$$

is $O\left(t^{2}\right)$ as $t$ tends to zero. Also, vectors in the cokernel (the kernel of the transpose matrix) correspond to self stresses. Moreover we have the following fundamental definition.

Definition 2.1. A framework $(G, p)$ in the plane (resp. in $\mathbb{R}^{3}$ ) with graph $G=(V, E)$ is infinitesimally rigid if the rank of $R(G, p)$ is $2|V|-$ 3 (resp. $3|V|-6)$ and is isostatic if it is infinitesimally rigid and the rank of $R(G, p)$ is $|E|$.

As an illustration we shall keep in view the symmetric framework $(G, p)$ in $\mathbb{R}^{2}$ indicated in Figure 1, with framework vector

$$
p=((2,0),(3,1),(4,0),(3,-1),(-4,0),(-3,1),(-2,0),(-3,-1)) .
$$

The subframework on the first four vertices is infinitesimally rigid as is its mirror image in the $y$-axis. The entire framework appears to have one non-trivial infinitesimal flex in addition to the three spatial flexes and this is readily confirmed.

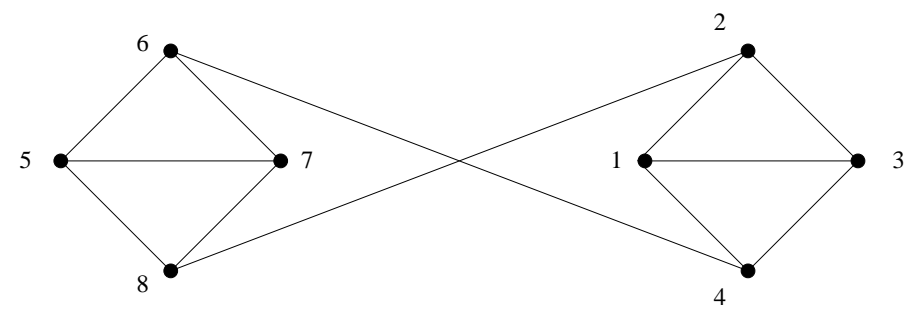

FiguRE 2. A symmetric bar-joint framework.

The rigidity matrix has the form

$$
R=\left[\begin{array}{cc}
R_{1} & 0 \\
0 & R_{1} \\
T_{1} & T_{2}
\end{array}\right]
$$


where $R_{1}$ is the 5 by 8 matrix

$$
R_{1}=\left[\begin{array}{cccccccc}
-1 & -1 & 1 & 1 & 0 & 0 & 0 & 0 \\
0 & 0 & -1 & 1 & 0 & 0 & 1 & -1 \\
0 & 0 & 0 & 0 & 1 & 1 & -1 & -1 \\
-1 & 1 & 0 & 0 & 0 & 0 & 1 & -1 \\
2 & 0 & 0 & 0 & -2 & 0 & 0 & 0
\end{array}\right]
$$

and where the submatrix $\left[\begin{array}{ll}T_{1} & T_{2}\end{array}\right]$ is the 2 by 16 matrix corresponding to the two long framework edges $\left[p_{2}, p_{8}\right],\left[p_{4}, p_{6}\right]$.

2.2. Graph symmetry. Let $G$ have vertices $v_{1}, \ldots, v_{n}$ and let $\sigma$ be a permutation of $(1, \ldots, n)$ corresponding to an automorphism of $G$. We also write $\sigma: V \rightarrow V$ and $\sigma: E \rightarrow E$ for the corresponding bijective maps so that $\sigma\left(v_{i}\right)=v_{\sigma(i)}$. Let $\sigma_{e}$ denote the associated linear transformation of $\mathcal{H}_{e}$, where $\sigma_{e} \xi_{f}=\xi_{\sigma(f)}$, and let $\sigma_{e}$ also denote its representing matrix. The transformation and matrix $\sigma_{v}$ is similarly defined on the space $\mathcal{H}_{v}$ by the specification $\sigma_{v} \xi_{x_{i}}=\xi_{x_{\sigma(i)}}, \sigma_{v} \xi_{y_{i}}=$ $\xi_{y_{\sigma(i)}}, 1 \leq i \leq n$.

We first note how $R(G, p)$ is transformed, even in the absence of framework symmetry, on replacing the framework vector $p=\left(p_{1}, \ldots, p_{n}\right)$ by $\sigma(p)=\left(p_{\sigma(1)}, \ldots, p_{\sigma(n)}\right)$.

Lemma 2.2. Let $(G, p)$ be a framework in $\mathbb{R}^{d}$ with rigidity matrix $R(G, p)$ and let $\sigma$ be a graph automorphism. Then

$$
R(G, \sigma(p))=\sigma_{e}^{-1} R(G, p) \sigma_{v}
$$

Proof. For notational simplicity let $d=2$. Let $\sigma(p)=\left(p_{\sigma(1)}, \ldots, p_{\sigma(n)}\right)=$ $\left(p_{1}^{\prime}, \ldots, p_{n}^{\prime}\right)$, and $p_{i}^{\prime}=\left(x_{i}^{\prime}, y_{i}^{\prime}\right), 1 \leq i \leq n$. Associated with $e=\left(v_{i}, v_{j}\right)$ we have $x_{i}^{\prime}-x_{j}^{\prime}=x_{\sigma(i)}-x_{\sigma(j)}$. This difference appears in the $\sigma(e)$ row and $\sigma\left(x_{i}\right)$ column of $R(G, p)$ and so

$$
x_{i}^{\prime}-x_{j}^{\prime}=\left\langle R(G, p) \xi_{\sigma\left(x_{i}\right)}, \xi_{\sigma(e)}\right\rangle .
$$

On the other hand, from the definition of $R(G, \sigma(p))$,

$$
\begin{aligned}
x_{i}^{\prime}-x_{j}^{\prime} & =\left\langle R(G, \sigma(p)) \xi_{x_{i}}, \xi_{e}\right\rangle \\
& =\left\langle R(G, \sigma(p)) \sigma_{v}^{-1} \xi_{\sigma\left(x_{i}\right)}, \sigma_{e}^{-1} \xi_{\sigma(e)}\right\rangle \\
& =\left\langle\sigma_{e} R(G, \sigma(p)) \sigma_{v}^{-1} \xi_{\sigma\left(x_{i}\right)}, \xi_{\sigma(e)}\right\rangle
\end{aligned}
$$

and so $R(G, p)$ and $\sigma_{e} R(G, \sigma(p)) \sigma_{v}^{-1}$ have the same entry in the $\sigma(e)$ row and $\sigma\left(x_{i}\right)$ column. Similarly, all entries agree.

2.3. Framework symmetries. Let $(G, p)$ be a framework in $\mathbb{R}^{d}$ which is proper in the sense that the framework points are all distinct. Then a framework symmetry is a graph automorphism $\sigma$ of $G$ with the additional property

$$
\left|p_{\sigma(i)}-p_{\sigma(j)}\right|=\left|p_{i}-p_{j}\right|
$$


for all edges $\left(v_{i}, v_{j}\right)$, where $\left|p_{i}-p_{j}\right|$ denotes Euclidean distance. Note that such a symmetry may just act locally. The framework of Figure 1 for example has such a symmetry which exchanges $p_{1}$ and $p_{3}$. We shall mainly be concerned with the stricter global symmetries of frameworks that are determined by isometric maps of the ambient Euclidean space. Thus we formally define a spatial symmetry of $(G, p)$ as a framework symmetry which is effected by an isometric map $T: R^{d} \rightarrow \mathbb{R}^{d}$ in the sense that

$$
\sigma(p)=T p:=\left(T p_{1}, \ldots, T p_{n}\right)
$$

and we let $\mathcal{G}$ denote the spatial symmetry group of all such symmetries. In the final section however we shall relax this and consider spatial symmetries in subframeworks, and also latent spatial symmetries that appear after a partitioning.

The framework of Figure 1 has two evident mirror symmetries which are spatial symmetries and $\mathcal{G}$ is isomorphic to the four group $C_{2} \times C_{2}$.

Recall that an isometric map $T$ admits a factorisation as a product $T=T_{1} S T_{2}$, where $T_{1}, T_{2}$ are translations and $S$ is a linear isometry. The linearity of the entries in the rigidity matrix ensures that $R(G, p)=R(G, X p)$ if $X$ is a translation, and it follows that $R(G, T p)$ $=R(G, S p)$. Consider $S$ also in terms of the $d \times d$ real orthogonal matrix which effects the transformation $p_{i} \rightarrow S p_{i}$ by right matrix multiplication. In fact this matrix is $S^{-1}$ (where $S$ denotes also the matrix that effects the transformation $S$ ). For example, in case $d=2$, writing $\left(x_{i}^{\prime}, y_{i}^{\prime}\right)$ for the image $S p_{i}$ of $p_{i}$ under $S$, we have

$$
\left[\begin{array}{ll}
x_{i}^{\prime} & y_{i}^{\prime}
\end{array}\right]=\left[\begin{array}{ll}
x_{i} & y_{i}
\end{array}\right] S^{-1} .
$$

It follows from linearity that

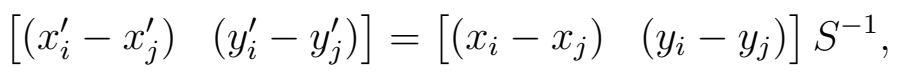

and so

$$
R(G, \sigma(p))=R(G, T p)=R(G, S p)=R(G, p) \tilde{S}^{-1}
$$

where $\tilde{S}=S \oplus \cdots \oplus S$ is the block diagonal matrix transformation of $\mathcal{H}_{v}$.

We now have all the ingredients for the proof of the individual symmetry equation of part (i) of Theorem 2.3. For the general formula of part (ii) we now specify five representations of the spatial symmetry group $\mathcal{G}$.

Write $\rho_{e}: \mathcal{G} \rightarrow \mathcal{L}\left(\mathcal{H}_{e}\right)$ for the permutation representation of $\mathcal{G}$ where $\rho_{e}(g)$ is the transformation and the matrix which is associated as above with the spatial symmetry $g$. Define $\rho_{v}: \mathcal{G} \rightarrow \mathcal{L}\left(\mathcal{H}_{v}\right)$ similarly. Let $\rho_{s p}: \mathcal{G} \rightarrow \mathcal{L}\left(\mathbb{R}^{d}\right)$ be the orthogonal group representation (one often identifies $\mathcal{G}$ with its image under this map) and let $\tilde{\rho}_{s p}=\rho_{s p} \oplus \cdots \oplus \rho_{s p}$ ( $n$ times) be the associated block diagonal representation of $\mathcal{G}$ on $\mathcal{H}_{v}$. 
Finally, note that $\tilde{\rho}_{s p}$ and $\rho_{v}$ commute, that is,

$$
\tilde{\rho}_{s p}\left(g_{1}\right) \rho_{v}\left(g_{2}\right)=\rho_{v}\left(g_{2}\right) \tilde{\rho}_{s p}\left(g_{1}\right)
$$

for all $g_{1}, g_{2}$. Thus the product representation, denoted $\hat{\rho}_{v}$, is welldefined. Indeed, these representations can be viewed as representations in different factors of the natural tensor product identification $\mathcal{H}_{v}=\mathbb{R}^{n} \otimes \mathbb{R}^{d}$ and $\hat{\rho}_{v}=\rho_{n} \otimes \rho_{s p}$, where $\rho_{n}$ is the (multiplicity one) representation for the vertices, so that $\rho_{v}=\rho_{n} \otimes I d_{d}$, and $\tilde{\rho}_{s p}=I d_{n} \otimes \rho_{s p}$, where $I d_{n}$ denotes the identity representation of multiplicity $n$.

The next theorem provides symmetry equations for the rigidity matrix. For an alternative somewhat more sophisticated derivation one may employ the chain rule for the derivative of composite multi-variable functions and we do this in Section 4 in a more abstract setting.

Theorem 2.3. Let $(G, p)$ be a framework in $\mathbb{R}^{d}$ with graph $G=(V, E)$.

(i) If $T$ is a spatial symmetry for the framework $(G, p)$ with associated graph symmetry $\sigma: V \rightarrow V$ and linear transformation matrices $\sigma_{v}$ and $\sigma_{e}$ then

$$
R(G, p)=\sigma_{e}^{-1} R(G, p) \sigma_{v} \tilde{S}
$$

where $S$ is the linear isometry factor of $T$ and $\tilde{S}=S \oplus \cdots \oplus S$ is the induced operator on $\mathcal{H}_{v}$.

(ii) Let $\mathcal{G}$ be the spatial symmetry group of the framework $(G, p)$ with representation $\hat{\rho}_{v}=\rho_{n} \otimes \rho_{s p}$ on $\mathcal{H}_{v}$ and representation $\rho_{e}$ on $\mathcal{H}_{e}$. Then, for all $g \in \mathcal{G}$,

$$
R(G, p)=\rho_{e}\left(g^{-1}\right) R(G, p) \hat{\rho}_{v}(g) .
$$

Proof. We may combine the equations 2.1 and 2.2 to obtain

$$
\sigma_{e}^{-1} R(G, p) \sigma_{v}=R(G, p) \tilde{S}^{-1},
$$

from which (i) follows. Now (ii) follows from (i) and the definition of the representations $\rho_{e}$ and $\hat{\rho}_{v}(g)$.

We note some immediate consequences for rigidity and isostaticity.

The analysis above applies also to what one might call grounded or supported frameworks $\left(G, p^{*}\right)$ in which certain vertices are fixed absolutely. The relevant symmetries in this case permute these special points. Such examples can be found in the original three-pointsupported symmetric two-dimensional structures in Kangwai and Guest[4] and Fowler and Guest[2].

The context is simpler since spatial flexes are absent and isostaticity of the suspended framework corresponds to the invertibility of the Jacobian $J\left(G, p^{*}\right)$ for the equation system for the free points. The argument for Theorem 2.2 (ii) applies and we obtain

$$
J\left(G, p^{*}\right)=\rho_{e}\left(g^{-1}\right) J\left(G, p^{*}\right) \hat{\rho}_{v}(g),
$$



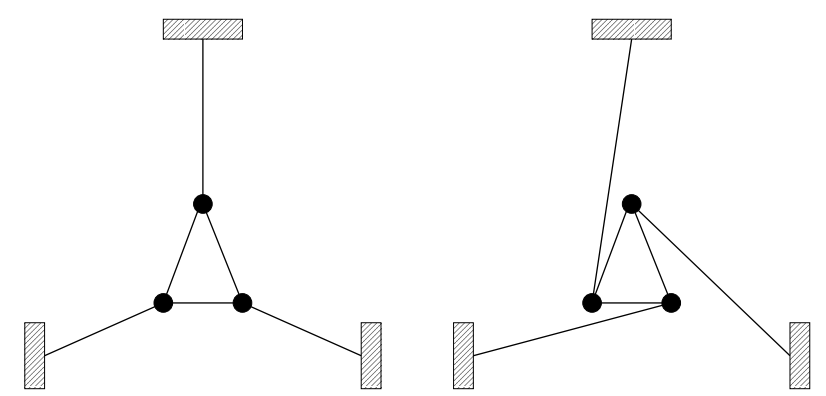

FiguRE 3. The singular Jacobian for the first framework is a consequence of reflection symmetry.

which is valid for elements $g$ of the spatial symmetry group $\mathcal{G}$, where $\rho_{v}$ is the representation for free vertices. In particular if $\left(G, p^{*}\right)$ is isostatic then

$$
\rho_{e}(g)=J\left(G, p^{*}\right) \hat{\rho}_{v}(g) J\left(G, p^{*}\right)^{-1}
$$

and so we obtain the following equalities of traces (also called characters); for each spatial symmetry group element $g$,

$$
\begin{aligned}
\operatorname{trace}\left(\rho_{e}(g)\right) & =\operatorname{trace}\left(\hat{\rho}_{v}(g)\right) \\
& =\operatorname{trace}\left(\rho_{n}(g) \otimes \rho_{s p}(g)\right) \\
& =\operatorname{trace}\left(\rho_{n}(g)\right) \operatorname{trace}\left(\rho_{s p}(g)\right) .
\end{aligned}
$$

For the identity symmetry element one obtains the simple counting condition $e^{\prime}=2 v^{\prime}$, where $e^{\prime}$ is the number of bars and $v^{\prime}$ is the number of free joints. If a reflection symmetry $g=\sigma$ exists then since $\operatorname{trace}\left(\rho_{s p}(\sigma)\right)=0$ one obtains trace $\left(\rho_{e}(\sigma)\right)=0$ which is to say that there can be no edges that are left fixed by the reflection.

As an illustration, consider the bilaterally symmetric frameworks of Figure 2. From the above it follows that $(G, p)$ is not isostatic if there is a reflection symmetry of the framework which leaves invariant at least one edge. In this manner the symmetry equation serves as a device for recognising singular systems which is somewhat simpler than the full Fowler-Guest equation.

\section{Flexes, Stresses and the Fowler-Guest Formula}

Let $(G, p)$ be a proper framework in $\mathbb{R}^{d}$, that is, one with distinct framework points, and let $\mathcal{H}_{f l}=\operatorname{ker} R(G, p)$ and let $\mathcal{H}_{s t}=\operatorname{ker} R(G, p)^{*}$ denote the kernel (nullspace) of the adjoint (conjugate transpose) matrix. The notation reflects the fact that the vectors of $\mathcal{H}_{f l}$ can be interpreted as infinitesimal flexes of the framework and that the vectors of $\mathcal{H}_{s t}$ represent self stresses of the framework, as we have indicated above. In fact the infinitesimal flexes are the vectors in the kernel of the derivative of the nonlinear mapping from framework points coordinates to framework edge lengths. This derivative, as we have noted is twice the rigidity matrix. 
The symmetry equation shows immediately the key fact that for all $g \in \mathcal{G}$

$$
\hat{\rho}_{v}(g) \mathcal{H}_{f l}=\mathcal{H}_{f l}, \rho_{e}(g) \mathcal{H}_{s t}=\mathcal{H}_{s t} .
$$

That is, that these spaces are invariant subspaces for the representations. Thus with respect to the orthogonal decompositions $\mathcal{H}_{v}=$ $\mathcal{H}_{v^{\prime}} \oplus \mathcal{H}_{f l}$,

$\mathcal{H}_{e}=\mathcal{H}_{e^{\prime}} \oplus \mathcal{H}_{s t}$ the matrix $R$ takes the block form

$$
R=\left[\begin{array}{cc}
R^{\prime} & 0 \\
0 & 0
\end{array}\right]
$$

where $R^{\prime}$ has trivial kernel and maps $\mathcal{H}_{v^{\prime}}$ onto $\mathcal{H}_{e^{\prime}}$. Certainly $\mathcal{H}_{f l}$ is nonzero since it contains the space, $\mathcal{H}_{\text {rig }}$ say, corresponding to ambient rigid body motion. In the case $d=2$ one may take as a basis for $\mathcal{H}_{\text {rig }}$ the vectors

$$
u_{x}=(1,0,1,0, \ldots, 1,0), u_{y}=(0,1,0,1, \ldots, 0,1),
$$

(which are associated with infinitesimal translation), together with the vector $u_{x y}$ (associated an infinitesimal rotation about the origin) given by

$$
u_{x y}=\left(-y_{1}, x_{1},-y_{2}, x_{2}, \ldots,-y_{n}, x_{n}\right),
$$

where $\left(x_{i}, y_{i}\right)$ are the coordinates of the framework points $p_{i}$. In fact for the associated three dimensional subspace

$$
\mathcal{H}_{\text {rig }}=\mathcal{H}_{x} \oplus \mathcal{H}_{y} \oplus \mathcal{H}_{x y}
$$

the subrepresentation $\rho_{\text {rig }}$ of $\hat{\rho}_{v}$ (obtained by restriction to $\mathcal{H}_{\text {rig }}$ ) decomposes as 3 copies of the trivial one-dimensional representation.

Finally, define $\mathcal{H}_{\text {mech }}$ as the complementary space to $\mathcal{H}_{\text {rig }}$ in $\mathcal{H}_{f l}$, so that $\mathcal{H}_{f l}=\mathcal{H}_{\text {mech }} \oplus \mathcal{H}_{\text {rig }}$. The notation reflects the fact that this subspace may be viewed as the space for non-trivial infinitesimal motions (mechanisms) of the framework.

With these Euclidean space decompositions, which are all in terms of invariant subspaces for $\hat{\rho}_{v}$ ), we have the associated decompositions

$$
\hat{\rho}_{v}=\rho_{v^{\prime}} \oplus \rho_{f l}=\rho_{v^{\prime}} \oplus \rho_{\text {mech }} \oplus \rho_{\text {rig }} .
$$

For the other representation $\rho_{e}$ we have the two-fold decomposition

$$
\rho_{e}=\rho_{e^{\prime}} \oplus \rho_{s t}
$$

associated with the orthogonal decomposition $\mathcal{H}_{e}=\mathcal{H}_{e^{\prime}} \oplus \mathcal{H}_{s t}$.

We can now give a complete proof of a general form of the FowlerGuest formula 1.2. In brief, the formula follows immediately from the similarity (and unitary equivalence) of the "residual" representations $\rho_{v^{\prime}}$ and $\rho_{e^{\prime}}$ following the removal of subrepresentations corresponding to flexes (both ambient and nontrivial) and to stresses, and this similarity follows from the symmetry equation for the rigidity matrix. 
Write $\left[\rho_{x}\right]$ to denote the character list of a representation $\rho_{x}$. Explicitly, this is the list $\left(\operatorname{trace}\left(\rho_{x}\left(g_{1}\right)\right), \ldots, \operatorname{trace}\left(\rho_{x}\left(g_{N}\right)\right)\right)$ for some enumeration of the elements (or the generators) of $\mathcal{G}$.

Theorem 3.1. Let $(G, p)$ be a bar-joint framework in $\mathbb{R}^{d}$, with $n$ distinct joints and $m$ bars, and with spatial symmetry group $\mathcal{G}$ with orthogonal representation $\rho_{s p}$ in $\mathbb{R}^{d}$. Let $\rho_{n}, \rho_{e}$ be the joint and bar (permutation) representations of $\mathcal{G}$ on $\mathbb{R}^{n}$ and $\mathbb{R}^{m}$ respectively, let $\rho_{\text {rig }}$ be the subrepresentation of $\rho_{n} \otimes \rho_{\text {sp }}$ for the space of trivial infinitesimal flexes, let $\rho_{\text {mech }}$ be the subrepresentation for nontrivial flexes, and let $\rho_{s t}$ be the subrepresentation of $\rho_{e}$ for the space of internal stresses. Then

$$
\left[\rho_{\text {mech }}\right]-\left[\rho_{s t}\right]=\left[\rho_{n}\right] \cdot\left[\rho_{s p}\right]-\left[\rho_{e}\right]-\left[\rho_{\text {rig }}\right]
$$

where [ ] [ [ ] denotes entry-wise product of characters.

Proof. Recall that with respect to the orthogonal decomposition

$$
\mathcal{H}_{v}=\mathcal{H}_{v}^{\prime} \oplus \mathcal{H}_{f l}, \mathcal{H}_{e}=\mathcal{H}_{e}^{\prime} \oplus \mathcal{H}_{s t}
$$

the rigidity matrix $R$ takes the block form

$$
R=\left[\begin{array}{cc}
R^{\prime} & 0 \\
0 & 0
\end{array}\right]
$$

The matrix $R^{\prime}$ is a square nonsingular matrix which we view as a linear transformation $R^{\prime}: \mathcal{H}_{v}^{\prime} \rightarrow \mathcal{H}_{e}^{\prime}$. From the symmetry equation we have the commutation relations $R^{\prime} \rho_{v^{\prime}}(g)=\rho_{e^{\prime}}(g) R^{\prime}$ and so

$$
\operatorname{trace}\left(\rho_{v^{\prime}}(g)\right)=\operatorname{trace}\left(\left(R^{\prime}\right)^{-1}\right) \rho_{e^{\prime}}(g) R^{\prime}=\operatorname{trace}\left(\rho_{e^{\prime}}(g)\right) .
$$

Thus $\rho_{v^{\prime}}$ and $\rho_{e^{\prime}}$ have the same character list; $\left[\rho_{v^{\prime}}\right]=\left[\rho_{e^{\prime}}\right]$.

We have

$$
\left[\rho_{n}\right] \cdot\left[\rho_{s p}\right]=\left[\rho_{n} \otimes \rho_{s p}\right]=\left[\rho_{v^{\prime}}\right]+\left[\rho_{\text {mech }}\right]+\left[\rho_{\text {rig }}\right]
$$

and

$$
\left[\rho_{e}\right]=\left[\rho_{e}^{\prime}\right]+\left[\rho_{s t}\right]
$$

and so from $\left[\rho_{v^{\prime}}\right]=\left[\rho_{e^{\prime}}\right]$ we obtain equation 3.3.

We note that one can also make explicit an orthogonal equivalence between the residual representations $\rho_{v^{\prime}}, \rho_{e^{\prime}}$ through the isometric part $U$ of the polar decomposition $R^{\prime}=U\left|R^{\prime}\right|$ as this operator also intertwines the representations; $U \rho_{v^{\prime}}(g)=\rho_{e^{\prime}}(g) U$, for $g \in \mathcal{G}$.

The right hand side of the Fowler-Guest formula is readily computable in terms of the number of elements fixed by a framework symmetry. Thus one may quickly obtain necessary counting conditions for such elements when the number of independent stresses and mechanisms are specified. Recall that a framework $(G, p)$ is isostatic if it is infinitesimally rigid and is stress free. Thus, in the case of planar 
isostatic framework if there is a mirror symmetry $\sigma$ evaluation of the formula at $\sigma$ gives

$$
0-0=\operatorname{trace}\left(\rho_{n}(\sigma)\right) \operatorname{trace}\left(\rho_{s p}(\sigma)\right)-\operatorname{trace}\left(\rho_{e}(\sigma)\right)-\operatorname{trace}\left(\rho_{\text {rig }}(\sigma) .\right.
$$

Since $\operatorname{trace}\left(\rho_{s p}(\sigma)\right)=0$ and $\operatorname{trace}\left(\rho_{\text {rig }}(\sigma)\right)=1$ we obtain

$$
0=0-b_{\sigma}+1
$$

where here we follow Connelly et al[7] and write $j_{\sigma}$ and $b_{\sigma}$ for the number of framework points (joints) and framework edges (bars) that are not displaced by $\sigma$.

Let us consider the framework of Figure 1 once more. Adding a cross edge between one of $p_{1}, p_{2}, p_{3}, p_{4}$ and one of $p_{5}, p_{6}, p_{7}, p_{8}$ will create realisations of a Laman graph. Addition of $\left[p_{2}, p_{6}\right]$ removes both mirror symmetries, so the count condition above is irrelevant and indeed the framework is isostatic. Also note that addition of $\left[p_{2}, p_{6}\right]$ is consistent with the necessary condition for the one remaining mirror symmetry. On the other hand addition of an edge on the $x$-axis violates the count $b_{\sigma}=1$, for both mirror symmetries, and for this reason the resulting framework is not isostatic.

We can obtain also the following corollary which is indicative of the results obtained in Connelly et al[7].

Corollary 3.2. Let $(G, p)$ be an isostatic framework in $\mathbb{R}^{3}$ which does not lie in a hyperplane and which has a proper spatial symmetry $\sigma$. Then the following equations hold.

(i) If $\sigma$ is a half turn then $0=-j_{\sigma}-b_{\sigma}+2$.

(ii) If $\sigma$ is a reflection then $0=j_{\sigma}-b_{\sigma}$.

(iii) If $\sigma$ is an inversion then $0=-3 j_{\sigma}-b_{\sigma}$.

Proof. (i) In this case trace $\left(\rho_{n}(\sigma)\right)=j_{\sigma}$ since $\rho_{n}(\sigma)$ is a permutation matrix with a nonzero diagonal entry if and only if the corresponding vertex is fixed by $\sigma$. Also trace $\left(\rho_{s p}(\sigma)\right)=-1$, since $\rho_{s p}(\sigma)$ is equivalent to a diagonal matrix with entries $-1,-1,1$, and $\rho_{\text {rig }}(\sigma)=-2$ since in the three dimensional subspace for infinitesimal translation flexes $\rho_{\text {rig }}(\sigma)$ is diagonal with entries $-1,-1,1$, and in the three dimensional subspace for infinitesimal rotation flexes $\rho_{\text {rig }}(\sigma)$ is similarly diagonal with entries $-1,-1,1$.

From these observations and the previous character formula, evaluated at $\sigma$, statement (i) follows. The formulae of (ii) and (iii) are similarly verified; in case (ii), $\operatorname{trace}\left(\rho_{s p}(\sigma)\right)=1, \operatorname{trace}\left(\rho_{\text {rig }}(\sigma)\right)=0$ and in case (iii), trace $\left(\rho_{s p}(\sigma)\right)=-3$ and $\operatorname{trace}\left(\rho_{\text {rig }}(\sigma)\right)=0$.

\section{Higher Order Frameworks and Symmetry}

We now show how the approach above adapts readily to higher dimensional frameworks such as point-line frameworks in $\mathbb{R}^{2}$, body-bar frameworks in $\mathbb{R}^{3}$, and even infinite frameworks. 
4.1. Character formulae for point-line frameworks. Consider, in $\mathbb{R}^{2}$, a set $\mathcal{P}$ of points and a set $\mathcal{L}$ of straight lines,

$$
\mathcal{P}=\left\{p_{1}, \ldots, p_{n}\right\}, \quad \mathcal{L}=\left\{L_{1}, \ldots, L_{r}\right\} .
$$

Considering only certain pairs from $\mathcal{P} \cup \mathcal{L}$ we can compute generalised distances involving the lines, namely point-line distances, being the usual nonnegative distance, and line-line angles, taking values in $[0, \pi / 2]$. The chosen pairs determine edges $e \in E$ in an abstract graph whose vertex set $V$ is partitioned $V=V_{p} \cup V_{l}$ and whose edge set is similarly partitioned, $E=E_{p p} \cup E_{p l} \cup E_{l l}$. The abstract partitioned graph $G$ and the pair $\mathcal{P}, \mathcal{L}$ give rise to a distance labeled graph. This is the pair $(G, d)$ where $d$ is a map from $E$ to the set of distances; $d(e)=d\left(p_{i}, p_{j}\right)$, for $e=(i, j) \in E_{p p}, d(e)=d\left(p_{i}, L_{j}\right)$, for $e \in E_{p l}$ and $d(e)=d\left(L_{i}, L_{j}\right)$ for $e=(i, j) \in E_{l l}$.

It is of interest to understand the inverse problem, that is, the nature of solutions of the constraint equations determined by an abstract distance labeled partitioned graph. These equations are in the coordinate variables for the points and lines. The points are coordinatised as usual, with variables $x_{i}, y_{i}$ for the framework point $p_{i}$. We may assume by translating that the lines $L_{j}$ do not pass through the origin and so may be parameterized by their closest points $\left(x_{j}^{\prime}, y_{j}^{\prime}\right)$ to the origin. Writing $\underline{x}$ for the set of all variables, this system can be indicated as the equation set

$$
f_{e}(\underline{x})=d(e), \quad e \in E .
$$

Let $(G, \mathcal{P}, \mathcal{L})$ be a point-line framework as above. Define $\mathcal{H}_{e}$ and $\mathcal{H}_{v}$ as before but with the natural additional structure:

$$
\mathcal{H}_{v}=\mathcal{H}_{p} \oplus \mathcal{H}_{l}
$$

and, according to edge type,

$$
\mathcal{H}_{e}=\mathcal{H}_{p p} \oplus \mathcal{H}_{p l} \oplus \mathcal{H}_{l l} .
$$

Also,

$$
\mathcal{H}_{p}=\sum_{k=1}^{n} \oplus\left(\mathbb{R}_{x_{k}} \oplus \mathbb{R}_{y_{k}}\right), \quad \mathcal{H}_{l}=\sum_{k=n+1}^{n+r} \oplus\left(\mathbb{R}_{x_{k}^{\prime}} \oplus \mathbb{R}_{y_{k}^{\prime}}\right) .
$$

We define the rigidity matrix for a line-plane framework, or a dimensioned abstract graph, simply as the Jacobian of the distance constraint equation system. The Jacobian has a $3 \times 2$ block structure implied by the vector space decompositions and takes the form,

$$
R(G, \mathcal{P}, \mathcal{L})=\left[\begin{array}{cc}
R(G, \mathcal{P}) & 0 \\
0 & R(G, \mathcal{L}) \\
R_{1} & R_{2}
\end{array}\right]
$$

and the representations $\rho_{e}$ and $\hat{\rho}_{v}$ have a corresponding three-fold and two-fold diagonal block structure, respectively. 
As before we have a spatial symmetry group $\mathcal{G}$ for the point-line framework $(G, \mathcal{P}, \mathcal{L})$. For simplicity we assume that the framework contains lines and points, that $0 \in \mathbb{R}^{2}$ is the centre of symmetry and that there are no lines through the origin. As before we have five representations :

$$
\rho_{e}, \rho_{v}, \rho_{s p}, \tilde{\rho}_{s p} \text { and } \hat{\rho}_{v}=\rho_{n} \otimes \rho_{s p} .
$$

Note in particular that the coordinates for the lines are analogous to the coordinates for points in that for a point-line framework symmetry $g$, given by a linear isometric transformation $T$ of $\mathbb{R}^{2}$, the coordinates for the transformed line $T\left(L_{j}\right)$ are $T\left(x_{j}^{\prime}, y_{j}^{\prime}\right)$.

Define $\mathcal{H}_{s t}=\operatorname{coker} R(G, \mathcal{P}, \mathcal{L})$ and let $\operatorname{ker} R(G, \mathcal{P}, \mathcal{L})=\mathcal{H}_{\text {rig }} \oplus \mathcal{H}_{\text {mech }}$ where $\mathcal{H}_{\text {rig }}$ is the three dimensional space of infinitesimally rigid flexes. Thus the space $\mathcal{H}_{\text {mech }}$ is defined as the (possibly zero) orthogonal complement of $\mathcal{H}_{\text {rig }}$ in $\operatorname{ker} R(G, \mathcal{P}, \mathcal{L})$. The point-line framework is said to be isostatic if it is infinitesimally rigid, that is, if if $\mathcal{H}_{\text {mech }}=\{0\}$, and also that it is stress free in the sense that $\mathcal{H}_{s t}=\{0\}$.

Theorem 4.1. Let $(G, \mathcal{P}, \mathcal{L})$ be a point-line framework as above with spatial symmetry group $\mathcal{G}$. Then

$$
R(G, \mathcal{P}, \mathcal{L})=\rho_{e}\left(g^{-1}\right) R(G, \mathcal{P}, \mathcal{L}) \hat{\rho}_{v}(g), \quad g \in \mathcal{G},
$$

and, as an equality of character lists,

$$
\left[\rho_{\text {mech }}\right]-\left[\rho_{s t}\right]=\left[\rho_{n}\right] \cdot\left[\rho_{s p}\right]-\left[\rho_{e}\right]-\left[\rho_{\text {rig }}\right] .
$$

In particular if the framework is isostatic and has a proper reflection symmetry, with graph automorphism $\sigma \neq i d$, then

$$
b_{p p}+b_{l l}+b_{p l}-1=0
$$

where $b_{p p}, b_{l l}$ and $b_{p l}$ are the number of point-point edges, line-line edges and point-line edges which are unchanged by the reflection.

Proof. In the next subsection we obtain a general symmetry formula and the stated formula is a special case of this. The character list formula is proven in exactly the same manner as in the proof of Theorem 3.1

As we have noted in the introduction, this theorem can be useful for predicting the singularity of an equation system underlying a CAD diagram.

4.2. Higher order frameworks. We now derive symmetry equations for the rigidity matrix of quite general distance constrained systems using a more direct proof using the Jacobean derivative of the generalised edge map. A simple example of the abstract formulation below is the case of finite systems of points and (unoriented) planes in $\mathbb{R}^{3}$, with constraints of Euclidean distance between points, and points and planes, 
and with angular constraints between planes. Planes may be coordinatised by the three coordinates of the point closest to the origin and so play a role similar to points.

Let $(G, E)$ be a finite, connected, undirected graph and let $V=$ $V_{1} \cup \cdots \cup V_{n}$ be a partition in which $V_{i}=\left\{v_{i, k}: 1 \leq k \leq \nu_{i}\right\}$ is a set of vertices which label a set $\mathcal{P}_{i}=\left\{p_{i, k}: 1 \leq k \leq \nu_{i}\right\}$ of geometric objects of the same kind. Formally, each object of the $i^{t h}$ kind, $p_{i, k} \subseteq \mathbb{R}^{d}$, is a real manifold, or, more generally, a real semi-algebraic set, which is determined by a specification $\underline{x}_{i}=\left(x_{i, 1}, \ldots, x_{i, t_{i}}\right)$ of $t_{i}$ parameters. For example, a straight line in three dimensions requires four variables.

For a pair of specified objects $(p, q)$, either of the same or differing type, a generalised distance equation is given which has the form $f(p, q)=d$ where $d$ is real and $f$ is a function in the parameters for $p, q$. We say that the constraint is a Euclidean constraint if for all isometries of $\mathbb{R}^{d}$ and all objects $p, q$ of the appropriate type, we have $f(T p, T q)=f(p, q)$.

Definition 4.2. A Euclidean framework is a pair $(G, \mathcal{P})$ together with a family of distance functions $f_{e}(p, q), e \in E$ where

(i) $G=(V, E)$ is a graph with partitioned vertex set $V$ labeling a set $\mathcal{P}$ of specified objects, with objects of the same kind in each partition set, and

(ii) the distance functions $f_{e}(p, q)$ are Euclidean invariant and depend only on the type of the objects $p, q$.

To consider the rigidity or flexibility of a particular Euclidean framework $(G, \mathcal{P})$ we consider the framework equation system, which, by definition, is the constraint system

$$
f_{e}\left(\underline{x}_{i, k}, \underline{x}_{j, l}\right)=d_{e}, \quad e=\left(v_{i, k}, v_{j, l}\right) \in E,
$$

A proper Euclidean framework $(G, \mathcal{P})$ is one for which the objects do not all lie in a hyperplane. We say that a framework of this type is infinitesimally rigid if the Jacobean $J(G, \mathcal{P})$ of the constraint system has rank equal to $N-d(d+1) / 2$ where

$$
N=\nu_{1} t_{1}+\cdots+\nu_{n} t_{n}
$$

is the total number of variables. Also we say that $(G, \mathcal{P})$ is isostatic if in addition the rank is equal to $|E|$.

Let $(G, \mathcal{P})$ be a Euclidean framework with geometric objects $p_{1}, \ldots, p_{n}$. Following the terminology for frameworks we define the constraint function, or edge map, of $(G, \mathcal{P})$ to be the nonlinear function $f: \mathbb{R}^{N} \rightarrow \mathbb{R}^{m}$ with

$$
f(\underline{x})=\left(f_{e_{1}}(\underline{x}), \ldots, f_{e_{m}}(\underline{x})\right),
$$

where $m=|E|$. Here the $i^{\text {th }}$ constraint function for the edge $e_{i}$ depends on the variables $\underline{x}_{k}, \underline{x}_{l}$ for the objects $p_{k}, p_{l}$ associated with $e_{i}$. 
We have

$$
\mathcal{H}_{v}=\sum_{k=1}^{n} \sum_{i=1}^{\nu_{k}} \oplus \mathbb{R}^{t_{i}},
$$

as the vector space for coordinate variables and if $T$ is an isometric transformation of $\mathbb{R}^{d}$ then there is an associated block diagonal transformation

$$
\tilde{T}=\sum_{k=1}^{n} \sum_{i=1}^{\nu_{k}} \oplus T_{k},
$$

where each $T_{k}$ is the parameter transformation induced by $T$. In particular, if $\sigma$ is a spatial symmetry of $(G, \mathcal{P})$ which additionally is induced by a spatial isometry $T$ then we call $\tilde{T}$ the local symmetry transformation for $\sigma$.

Similarly we have the edge space $\mathcal{H}_{e}$ on which the spatial symmetries $g$ act a permutation transformations.

We now obtain the symmetry equation for the rigidity matrix of a Euclidean framework, defined here as Jacobean derivative $D(f)(\underline{x})$ of the constraint map evaluated at the framework coordinates to yield the matrix $J(G, \mathcal{P})$.

Theorem 4.3. Let $(G, \mathcal{P})$ be a Euclidean framework, with generalised distance equations $f_{e}(p, q)=d_{e}, e \in E$, where $p, q$ denote the parameters of the two geometric elements constrained by distance $d_{e}$, let $f: \mathbb{R}^{N} \rightarrow \mathbb{R}^{m}$ be the generalised constraint map and let $(\sigma, T)$ be a spatial symmetry of $(G, \mathcal{P})$. Then the rigidity matrix $J(G, \mathcal{P})$ satisfies the symmetry equation

$$
J(G, \mathcal{P})=\sigma_{e}^{-1} J(G, \mathcal{P}) \sigma_{v} \tilde{T}=\sigma_{e}^{-1} J(G, \mathcal{P}) \tilde{T} \sigma_{v} .
$$

where $\sigma_{v}$ and $\sigma_{e}$ are the induced permutation transformations of the vertex space $\mathcal{H}_{v}$ and the edge space $\mathcal{H}_{e}$ and where $\tilde{T}$ is the local symmetry transformation for $\sigma$.

Proof. Let $\sigma$ and $T$ be as above. Then from the graph symmetry $\sigma$ it follows, as in Lemma 2.2, that evaluating the Jacobian at $\sigma(x)$ gives the same matrix as corresponding row and column operations on the Jacobian, that is,

$$
D f(\sigma(\underline{x}))=\sigma_{e}^{-1} D f(\underline{x}) \sigma_{v} .
$$

On the other hand, by Euclidian invariance $f(\tilde{T} \underline{x})=f(\underline{x})$ for all values of the variables, and so by the chain rule,

$$
(D f)(\tilde{T} \underline{x}) \tilde{T}=D f(\underline{x}) .
$$

However, we have $\sigma(\underline{x})=\tilde{T} \underline{x}$ for the given framework coordinates and putting these fact together yields in this case

$$
D f(\underline{x}) \tilde{T}^{-1}=D f(\tilde{T} \underline{x})=D f(\sigma(\underline{x}))=\sigma_{e}^{-1} D f(\underline{x}) \sigma_{v},
$$

as required. 
4.3. Pin-jointed body frameworks. We now consider a generalisation of bar-joint frameworks by allowing the edges to be general rigid bodies which may then have more than 2 vertices. Informally this looks like a set of rigid bodies which are held together by a set of pins or hinges, each of which passes through two or more bodies. Note that bar-joint and body-bar frameworks are both special cases of pinjointed body frameworks. The discussion below is self-contained. For other information on body bar frameworks see Tay and Whiteley [12] and Jackson and Jordan[13]. We limit attention to pin-jointed body frameworks in $\mathbb{R}^{2}$.

Definition 4.4. A pin-jointed body framework is a pair $(\mathcal{S}, p)$ where $p=\left\{p_{i}\right\}$ is a set of points in $\mathbb{R}^{2}$ and $\mathcal{S}=\left\{S_{e}\right\}$ a collection of subsets of the points such that:

(i) every point is in at least two sets,

(ii) every set contains at least two points.

We also shorten the term to "body framework" and we call the sets $S_{e}$ "bodies". The labelling notation here reflects the special case of edges and we occasionally denote a set $S_{e_{i}}$ simply by $e_{i}$. Every body framework defines a bipartite graph $G=G(\mathcal{S})$ in which the points are the vertices of one partition and the bodies are the vertices of the other partition. The edges of $G$ represent the occurrence of a point in a body. Conversely a bipartite graph with minimum vertex degree greater than one defines a body framework.

A flex (or infinitesimal flex, or infinitesimal motion) of a body framework is an assignment of velocities $u_{i}$ to the points $p_{i}$ and an assignment of infinitesimal motions $\left(v_{e}, a_{e}\right)$ to the bodies such that for each body the velocities of its points are compatible with the rigid motion $\left(v_{e}, a_{e}\right)$ of the body. Here $v_{e} \in \mathbb{R}^{2}$ is the velocity of the centroid of the body $e$ and $a_{e} \in \mathbb{R}$ is its angular velocity, and the centroid is defined as $p_{e}=\frac{1}{\left|S_{e}\right|} \sum_{p_{i} \in S_{e}} p_{i}$. The compatibility condition is the equation

$$
u_{i}=v_{e}+a_{e}\left(p_{i}-p_{e}\right)^{\pi / 2},
$$

where $v^{\pi / 2}$ denotes the rotated vector $(-y, x)$ when $v=(x, y)$. Thus there are two linear equations for every occurrence of a point in a body, that is, for every edge of the bipartite graph. With the coordinate notation $u_{i}=\left(u_{i}(x), u_{i}(y)\right)$ they take the form.

$$
\begin{gathered}
u_{i}(x)-v_{e}(x)+a_{e}\left(p_{i}(y)-p_{e}(y)\right)=0, \\
u_{i}(y)-v_{e}(y)-a_{e}\left(p_{i}(x)-p_{e}(x)\right)=0 .
\end{gathered}
$$

Suppose now that there are $n$ points, $e$ bodies and $c$ point-body occurrences, that is, $n+e$ vertices and $c$ edges in $G(\mathcal{S})$. We define a $(2 n+3 e)$ by $2 c$ rigidity matrix $R=R(\mathcal{S}, p)$ as follows.

(i) $R$ has 2 columns for each point and 3 columns for each body.

(ii) $R$ has 2 rows for each point-body occurrence. 
(iii) The 2 by 5 submatrix for (i) and (ii) with appropriately labeled columns, takes the form

$$
\left[\begin{array}{ccccc}
u_{i}(x) & u_{i}(y) & v_{e}(x) & v_{e}(y) & a_{e} \\
1 & 0 & -1 & 0 & -\left(p_{i}(y)-p_{e}(y)\right) \\
0 & 1 & 0 & -1 & \left(p_{i}(x)-p_{e}(x)\right)
\end{array}\right]
$$

A body framework is infinitesimally rigid if it has no non-trivial flexes. As usual there is a three-dimensional space of trivial flexes and so infinitesimal rigidity corresponds to there being no other nonzero solutions to the compatibility equations. This is simply the condition $\operatorname{dim}(\operatorname{ker} R)=3$. We say that a body framework is isostatic if $2 c=2 n+3 e-3$ and $\operatorname{rank} R=2 c$.

Consider now the natural decompositions of the domain space and the codomain space for the rigidity matrix regarded as a linear transformation.

Let $p_{1}, \ldots, p_{r}$ be the pin points of $(\mathcal{S}, p)$ and let $e_{1}, \ldots, e_{s}$ be the bodies. Let $\mathcal{H}_{\text {dom }}=\mathcal{H}_{b} \oplus \mathcal{H}_{p}$, where

$$
\mathcal{H}_{b}=\mathcal{H}_{\text {body }} \otimes \mathbb{R}^{3}=\sum_{i=1}^{s} \oplus \mathbb{R}^{3}, \quad \mathcal{H}_{p}=\mathcal{H}_{\text {pin }} \otimes \mathbb{R}^{2}=\sum_{i=1}^{r} \oplus \mathbb{R}^{2},
$$

where the summands $\mathbb{R}^{2}$ represent the spaces of displacement velocities $u_{i}$ for $p_{i}$ and where the summands $\mathbb{R}^{3}$ are the spaces of body velocities $\left(v_{e}(x), v_{e}(y), a_{e}\right)$. Similarly, the codomain space for $R$ has the form

$$
\mathcal{H}_{\text {codom }}=\mathcal{H}_{\text {mem }} \otimes \mathbb{R}^{2}=\sum_{i=1}^{N} \oplus \mathbb{R}^{2}
$$

associated with the $N$ edges of the bipartite graph of $(\mathcal{S}, p)$, that is, with the membership conditions $p_{i} \in e_{j}$.

Let $\mathcal{G}=\mathcal{G}(\mathcal{S}, p)$ be the group of isometries $T$ of $\mathbb{R}^{2}$ that are bodyframework symmetries. Thus $T p_{i}=p_{\pi(i)}$ for some permutation $\pi$ of the pins, and $\pi$ respects bodies, that is, the set $\pi\left(e_{i}\right)$ is equal to $e_{\tau(i)}$ for some permutation $\tau$. In particular the pair $(\pi, \tau)$ gives an automorphism of the abstract bipartite graph of the body framework.

Once again we consider various natural representations of $\mathcal{G}$. First we have $\rho_{b}=\rho_{\text {body }} \otimes I d_{3}$ and $\rho_{p}=\rho_{\text {pin }} \otimes I d_{2}$, the (inflated) permutation representations of the spatial symmetry group $\mathcal{G}$ on $\mathcal{H}_{b}$ and $\mathcal{H}_{p}$ associated with $\pi$ and $\tau$ respectively. As before, let $\rho_{s p}$ be the spatial representation of $\mathcal{G}$ as orthogonal transformations of $\mathbb{R}^{2}$, and let $\rho_{s p}^{+}$ be the representation $\rho_{s p} \oplus \Delta$ on $\mathbb{R}^{3}$ where $\Delta$ is the one dimensional determinant representation. We then have the natural representation of $\mathcal{G}$ on $\mathcal{H}_{\text {dom }}$ given by

$$
\rho_{\text {dom }}:=\hat{\rho}_{b} \oplus \hat{\rho}_{p}:=\left(\rho_{\text {body }} \otimes \rho_{s p}^{+}\right) \oplus\left(\rho_{\text {pin }} \otimes \rho_{s p}\right)
$$


where $\rho_{\text {body }}$ and $\rho_{\text {pin }}$ are the basic permutation representations for bodies and for pins.

Secondly, there is a representation $\rho_{\text {codom }}=\rho_{\text {mem }} \otimes \rho_{s p}$ of $\mathcal{G}$ associated with the permutation representation $\rho_{\text {mem }}$ for the edges of the bipartitie graph

In view of the form of the 2 by 5 submatrices above direct calculation gives the symmetry equations

$$
R=\rho_{\text {codom }}\left(g^{-1}\right) R \rho_{\text {dom }}(g), \text { for } g \in \mathcal{G} .
$$

As before these equations give to the invariance of various subspaces under the representations $\rho_{\text {dom }}$ and $\rho_{\text {codom }} ; \rho_{\text {rig }}$ is the subrepresentation of $\rho_{\text {dom }}$ determined by restriction to the subspace $\mathcal{H}_{\text {rig }}$ of trivial rigid body motion flexes, $\rho_{\text {mech }}$ is determined by the restriction to $\mathcal{H}_{\text {mech }}:=$ $\operatorname{ker} R \ominus \mathcal{H}_{\text {rig }}$, and $\rho_{\text {st }}$ is the restriction of $\rho_{\text {codom }}$ to the (internal stress) subspace $\mathcal{H}_{s t}:=$ coker $R$.

Theorem 4.5. Let $(\mathcal{S}, p)$ be a body framework in $\mathbb{R}^{2}$ with spatial symmetry group $\mathcal{G}$. Then the representation character lists satisfy the equation

$$
\left[\rho_{\text {mech }}\right]-\left[\rho_{s t}\right]=\left[\rho_{s p}^{+}\right] \cdot\left[\rho_{\text {body }}\right]+\left[\rho_{s p}\right] \cdot\left[\rho_{\text {pin }}\right]-\left[\rho_{\text {codom }}\right]-\left[\rho_{\text {rig }}\right] .
$$

Proof. The restriction of $R$ to the subspace $\mathcal{H}_{\text {dom }} \ominus\left(\mathcal{H}_{\text {mech }} \oplus \mathcal{H}_{\text {rig }}\right)$ gives a linear bijection to $\mathcal{H}_{\text {codom }} \ominus \mathcal{H}_{\text {st }}$ and so the associated ("residual") representations are equivalent. The formula now follows, as in the proof of Theorem 3.1

As a corollary we see that if the body framework is isostatic and has a reflection symmetry $\sigma$ then

$$
0=n_{\text {body }}^{\sigma}-1
$$

where $n_{\text {body }}^{\sigma}$ is the number of bodies left unmoved by $\sigma$. Indeed this follows from evaluating the character list equation at $\sigma$, for we then have

as well as

$$
\operatorname{trace}\left(\rho_{s p}^{+}(\sigma)\right)=1, \operatorname{trace}\left(\rho_{\text {body }}(\sigma)\right)=n_{\text {body }}^{\sigma}
$$

$$
\operatorname{trace}\left(\rho_{s p}(\sigma)\right)=0, \operatorname{trace}\left(\rho_{\text {codom }}(\sigma)\right)=0, \text { and } \operatorname{trace}\left(\rho_{\text {rig }}(\sigma)\right)=-1 \text {. }
$$

4.4. Symmetry equations for infinite frameworks. In Owen and Power $[17,18,19]$ we have indicated some perspectives for a mathematical theory of infinite bar-joint frameworks. Part of the motivation for such a development also comes from materials analysis (Donev and Torquato [20]), the analysis of repetitive structures (Guest and Hutchison [21]) and from applications in chemistry (Ceulemans et al[22] and crystallography (Borcea and Streinu[23]). We now consider the rigidity matrix symmetry equations in this setting. In particular we give a Hilbert space variant of Theorem 3.1 for a natural notion of squaresummable isostaticity, and we give a Fowler-Guest formula for periodic 
frameworks. Of course a novelty for infinite frameworks is that the spatial symmetry group $\mathcal{G}$ can be infinite.

4.4.1. Infinite frameworks. Let $(G, p)$ be a countable (and nonfinite) bar-joint framework in $\mathbb{R}^{2}$ associated with a countable connected graph $G$, where the framework vector $p=\left(p_{1}, p_{2}, \ldots\right)$ has framework points $p_{i}$ in $\mathbb{R}^{2}$ indexed as usual by the vertices of $G$. The consideration of such infinite frameworks of a general character, without translation symmetries, was begun in Owen and Power[17]. Here the divergence of various notions of rigidity was indicated as well as forms of rigidity allied to operator interpretations of the rigidity matrix. This latter theme is developed further in Owen and Power[19]. In addition to tools from operator theory it seems that general notions from functional analysis (such as uniform convergence, compactness, aperiodicity) will become of relevance to the analysis of infinite framework deformability. For our present consideration we address only infinitesimal rigidity rather than continuous rigidity and so we need only restrict attention to the rigidity matrix and its interpretations as a linear transformation.

Define the rigidity matrix $R(G, p)$ as in Section 2.1, with the rows labeled by edges and the columns labeled by vertices (twice over, for $x$ and $y$ coordinates). Assume that each vertex has finite degree. This entails that each column of the matrix has finitely many nonzero entries. This rigidity matrix may be viewed as a linear transformation $T$ from the direct product vector space $\mathcal{H}_{v}=\Pi_{V} \mathbb{R}^{2}$ to the vector space $\mathcal{H}_{e}=\Pi_{E} \mathbb{R}$. Here the direct product notation $\Pi_{E} \mathbb{R}$ indicates the set of all real sequences indexed by the edges of $G$, with the usual vector space structure. The permutation representation $\hat{\rho_{v}}$ and $\rho_{e}$ are defined on the spaces $\mathcal{H}_{v}$ and $\mathcal{H}_{e}$, respectively, as before.

Theorem 4.6. Let $(G, p)$ be an infinite bar-joint framework in $\mathbb{R}^{d}$ with rigidity matrix transformation $R(G, p): \mathcal{H}_{v} \rightarrow \mathcal{H}_{e}$. Then

$$
R(G, p)=\rho_{e}\left(g^{-1}\right) R(G, p) \hat{\rho}_{v}(g), \quad g \in \mathcal{G} .
$$

Proof. The sparse nature of the matrix for $R(G, p)$ ensures that the various infinite sums implied by matrix multiplication are sums over finitely many nonzero terms. With this change only the proof follows that of Theorem 2.3.

Once again, we may choose three linearly independent vectors in the kernel of $T$ to span the linear subspace of rigid motion flexes associated with a three-dimensional space $\mathcal{H}_{\text {rig }}$ for translations and rotations.

It is also natural to consider $R(G, p)$ as a linear transformation between other smaller sequence spaces which are invariant for the representations, and in this case the symmetry equations will hold as above. For example, let $T_{0}$ be the restriction of $R(G, p)$ to the vector space direct sum, $\mathcal{H}_{0}=\Sigma_{V} \oplus \mathbb{R}^{2}$, which consists of finite linear combinations of the usual standard basis vectors $\left(\xi_{x_{i}}\right.$ and $\left.\xi_{y_{i}}, i=1,2, \ldots\right)$. These are 
the "finitely supported vectors", that is, the sequences $u=\left(u_{v}\right)_{v \in V}$ in $\mathcal{H}_{0}$ which have all but finitely many entries equal to zero. One may view the vector $u$ as an assignment of velocity vectors to a finite number of joints of the infinite framework and view $T_{0}$ and associated mathematical constructs as modeling a very large system and its finitely acting disturbances. Note that $T_{0}$ maps into $\Sigma_{E} \oplus \mathbb{R}$, in view of the finiteness of vertex degrees. Also note that the translation and rotation flexes do not lie in the domain of $T_{0}$. It is natural then to say that $(G, p)$ is finitely infinitesimally rigid if the kernel of $T_{0}$ is trivial. The regular square grid framework (with framework points $(i, j), i, j \in \mathbb{Z}$ ) has this property as do grid frameworks with more generic vertex locations. Indeed it is enough to show that for any finite large square grid there is no nonzero flex which assigns zero velocities to the boundary joints. In fact we say that this framework is finitely isostatic since in this case there are also no nontrivial finitely supported stresses (vectors in the cokernel).

One can also consider other less severe constraints on the domain space, that is, on the allowable velocity vectors and flexes $u$, such as boundedness (each domain vector $u$ is a bounded sequence), summability $\left(\sum_{v}\left|u_{v}\right|<\infty\right)$, or square summability $\left(\sum_{v}\left|u_{v}\right|^{2}<\infty\right)$.

Let us define a square-summably isostatic framework in $\mathbb{R}^{d}$ as one for which

(i) the rigidity matrix $R(G, p)$ determines a bounded Hilbert space operator $T(G, p)$ from the real Hilbert space $\mathcal{H}_{v}^{2}:=\ell^{2}(V) \otimes \mathbb{R}^{d}$ to the real Hilbert space $\mathcal{H}_{e}^{2}:=\ell^{2}(E)$,

(ii) the kernel and cokernel of $T(G, p)$ are the zero subspaces.

Once again, for the spatial symmetry group we have the representations $\hat{\rho}_{v}=\rho_{v} \otimes \rho_{s p}$, on $\mathcal{H}_{v}^{2}$ and $\rho_{e}$ on $\mathcal{H}_{e}^{2}$. The following proposition is an infinite framework generalisation of the unitary equivalence noted in the finite case for the residual representations of $\mathcal{G}$.

Proposition 4.7. Let $(G, p)$ be a square-summably isostatic framework in $\mathbb{R}^{d}$. Then $\hat{\rho_{v}}$ and $\rho_{e}$ are unitarily equivalent representations and in particular have the same irreducible components.

Proof. We use a standard argument to show that the unitary part of $T=T(G, p)$ implements the equivalence.

Since $(G, p)$ is square summably isostatic $T$ has a unique polar decomposition of the form $T=U|T|$ with $U$ unitary. We have $\rho_{e}(g) T=$ $T \hat{\rho}_{v}(g)$ for all $g$. Thus $\left(\rho_{e}(g) T\right)^{*}=\left(T \hat{\rho}_{v}(g)\right)^{*}$ and so $T^{*} \rho_{e}(g)^{*}=$ $\left(\hat{\rho}_{v}(g)\right)^{*} T^{*}$, that is $T^{*} \rho_{e}\left(g^{-1}\right)=\left(\hat{\rho}_{v}\left(g^{-1}\right)\right) T^{*}$. Restating this, $T^{*} \rho_{e}(g)=$ $\left(\hat{\rho}_{v}(g)\right) T^{*}$, for all $g$. Thus, suppressing some notation, $T^{*} T \hat{\rho}_{v}=T^{*} \rho_{e} T=$ $T^{*} T \hat{\rho}_{v}$. Since $T^{*} T$ commutes with $\hat{\rho}_{v}$ so too does its square root $|T|$. We have $\rho_{e} U|T|=U|T| \hat{\rho}_{v}=U \hat{\rho}_{v}|T|$ and it follows, since $|T|$ has dense range for example, that $\rho_{e} U=U \hat{\rho}_{v}$ as desired. 
4.4.2. Periodic frameworks. We now show how the arguments of Section 3 can be applied to obtain Fowler-Guest type formulae for periodic bar-joint frameworks in $\mathbb{R}^{d}$. The trace lists indicated in Theorem 4.8 are associated with finite-dimensional representations of a finite group quotient $\mathcal{G} / \mathcal{T}$ of the spatial symmetry group $\mathcal{G}$, as we describe below.

Let $(G, p)$ be a countably infinite framework in $\mathbb{R}^{d}$ with distinct framework points and with spatial symmetry group $\mathcal{G}$ which contains a subgroup $\mathcal{T}$ isomorphic to $\mathbb{Z}^{d}$ with $d$ independent generators $W_{1}, \ldots, W_{d}$. It is in this sense that the framework is periodic. We assume that the framework points are discrete in the sense that there are finitely many $\mathcal{T}$-orbits of framework points. With this condition it follows that $\mathcal{G}$ is a crystallographic group. We do not assume that $\mathcal{T}$ is the minimal such subgroup. In that case the quotient $\mathcal{G} / \mathcal{T}$ would be the associated point group of $\mathcal{G}$ but it is also of interest to consider periodicity with respect to periods greater that the minimal period.

Consider the finite-dimensional Euclidean spaces $\mathcal{H}_{v}^{p} \subseteq \mathcal{H}_{v}$ and $\mathcal{H}_{e}^{p} \subseteq$ $\mathcal{H}_{e}$ consisting of the vectors that are periodic with respect to $\mathcal{T}$. From the symmetry equations

$$
\rho_{e}\left(W_{i}\right) R(G, p)=R(G, p) \hat{\rho}_{v}\left(W_{i}\right), \quad i=1, \ldots, d,
$$

it follows readily that the rigidity matrix $R(G, p)$ determines a linear transformation $R^{(p)}$ from $H_{v}^{p}$ to $\mathcal{H}_{e}^{p}$. The space $\operatorname{ker} R^{(p)}$ is the space ker $R(G, p) \cap H_{v}^{p}$, which can be viewed as the space of periodic "infinitesimal" flexes for the framework $(G, p)$. Similarly the space coker $R^{(p)}$ is the space of periodic "infinitesimal" stresses. (Note that a rotation flex $u=\left(u_{v}\right)_{v \in V}$, which is in the kernel of $R(G, p)$, is not a bounded sequence.)

The representations $\hat{\rho}_{v}, \rho_{e}$ of $\mathcal{G}$ induce representation $\hat{\pi}_{v}, \pi_{e}$ of $\mathcal{G} / \mathcal{T}$ on the periodic vector spaces. Explicitly, if $w=\left(w_{f}\right)_{f \in E}$ is in $\mathcal{H}_{e}^{p}$ then $\pi_{e}(g+\mathcal{T})$ is well-defined by the equation

$$
\left(\pi_{e}(g+\mathcal{T}) w\right)_{f}=w_{\sigma^{-1}(f)} .
$$

The representation $\hat{\pi}_{v}$ is defined similarly and the tensor factorisation of $\hat{\pi}$ gives the tensor factorisation $\hat{\pi}_{v}=\pi_{n} \otimes \rho_{s p}$.

Since the rigidity matrix transformation $R(G, p)$ and the transformations $\hat{\rho}_{v}(h), \rho_{e}(h), h \in \mathcal{G}$, leave invariant the spaces of periodic vectors we obtain from the symmetry equations for $R(G, p)$ and $\hat{\rho}_{v}, \rho_{e}$ the induced symmetry equations

$$
\pi_{e}(h) R^{(p)}=R^{(p)} \hat{\pi}_{v}(h), \quad h \in \mathcal{G} / \mathcal{T} .
$$

As before, the representations $\hat{\pi}, \pi_{e}$ do not depend on metrical detail and character lists for them are readily computable in terms of fixed elements.

Following the argument in Section 3, consider the orthogonal decompositions

$$
\mathcal{H}_{v}^{p}=\mathcal{H}_{v^{\prime}}^{p} \oplus \mathcal{H}_{m}^{p} \oplus \mathcal{H}_{r i g}^{p}
$$


where $\mathcal{H}_{\text {rig }}^{p}=\mathcal{H}^{p} \cap \mathcal{H}_{\text {rig }}$ and $\mathcal{H}_{\text {mech }}^{p}$ is the complementary space of $\mathcal{H}_{\text {rig }}^{\text {per }}$ in $\operatorname{ker} R^{(p)}$, and $\mathcal{H}_{v^{\prime}}^{p}$ is the complementary space of ker $R^{(p)}$ in $\mathcal{H}_{v}^{p}$. The rotational rigid motion flexes are not periodic and so this intersection is a $d$-dimensional space corresponding to the translation flexes. Similarly we have the decomposition

$$
\mathcal{H}_{e}^{p}=\mathcal{H}_{e^{\prime}}^{p} \oplus \mathcal{H}_{s t r}^{p} .
$$

From the symmetry equations we see that the component spaces

$$
\mathcal{H}_{v^{\prime}}^{p}, \mathcal{H}_{m}^{p}, \mathcal{H}_{\text {rig }}^{p}
$$

are invariant for $\hat{\pi}_{v}$ and so define subrepresentations of $\hat{\pi}_{v}$ whose trace lists we shall denote as

$$
\Gamma_{p}\left(v^{\prime}\right), \Gamma_{p}(m), \Gamma_{p}(\text { rig }),
$$

Similarly for the two subrepresentations of $\pi_{e}$ we obtain the character lists

$$
\Gamma_{p}\left(e^{\prime}\right), \Gamma_{p}(s)
$$

All five lists correspond to some fixed suppressed set $h_{1}, \ldots, h_{s}$ of generating elements of $\mathcal{G} / \mathcal{T}$.

Theorem 4.8. Let $(G, p)$ be a discrete periodic framework in $\mathbb{R}^{d}$ with spatial symmetry group $\mathcal{G}$ and let $\mathcal{T} \subseteq \mathcal{G}$ be a full rank translation subgroup isomorphic to $\mathbb{Z}^{d}$. Then

$$
\Gamma_{p}(m)-\Gamma_{p}(s)=\Gamma_{p}(v) \cdot \Gamma(s p)-\Gamma_{p}(e)-\Gamma_{p}(r i g)
$$

where $\Gamma_{p}(m)$ (resp. $\Gamma_{p}(s)$ ) are character lists for the representation of the finite group $\mathcal{G} / \mathcal{T}$ in the space of periodic (proper infinitesimal) mechanisms (resp. the space of periodic stresses).

Proof. The transformation $R^{(p)}$ induces an equivalence of the representations which shows that $\Gamma_{p}\left(v^{\prime}\right)=\Gamma_{p}\left(e^{\prime}\right)$. Since

$$
\Gamma\left(\hat{\pi}_{v}\right)=\Gamma_{p}(v) \cdot \Gamma(s p)=\Gamma_{p}\left(v^{\prime}\right)+\Gamma_{p}(m)+\Gamma_{p}(r i g)
$$

and

$$
\Gamma\left(\pi_{e}\right)=\Gamma(e)=\Gamma_{p}\left(e^{\prime}\right)+\Gamma_{p}(s)
$$

equation (11) follows.

Remark 4.9. In the case of planar periodic frameworks evaluating at the identity matrix give a periodic Maxwell rule, namely

$$
m_{p}-s_{p}=2\left|V_{p}\right|-\left|E_{p}\right|-2
$$

where $m_{p}$ and $m_{s}$ are the dimension of the spaces of periodic infinitesimal mechanisms and stresses, respectively, and $\left|V_{p}\right|$ and $\left|E_{p}\right|$ are the number of $\mathcal{T}$-orbits of vertices and edges, respectively. In the periodic isostatic case $m_{p}=s_{p}=0$ (by definition) and we have the necessary condition $2\left|V_{p}\right|-\left|E_{p}\right|-2$. 
Periodic rigidity and isostaticity has been developed in interesting work of Ross[24] who has obtained a periodic version of Laman's theorem in the case that the vertices in a unit cell for $\mathcal{T}$ are generically located. We also note that Borcea and Streinu[23] have considered more general forms of deformability of periodic frameworks. See also Owen and Power[19].

\section{Symmetry in SUBFRAMEWORKS AND PARTITIONS}

We now show how latent symmetries can play a role in predicting the singularity of asymmetric frameworks.

5.1. Subframework symmetry. Let $(G, p)$ be a proper bar-joint framework in $\mathbb{R}^{2}$ with a subframework $(X, p)$, where $X$ is a subgraph of $G$ (with at least one edge). Here, and below, it is convenient to use the redundant notation $(X, p)$ with $p$ the full framework vector. The Fowler-Guest formula holds for $(X, p)$ and in our notation takes the form

$$
\left[\rho_{\text {mech }}^{X}\right]-\left[\rho_{s t}^{X}\right]=\left[\rho_{s p}^{X}\right] \cdot\left[\rho_{n}^{X}\right]-\left[\rho_{e}^{X}\right]-\left[\rho_{\text {rig }}^{X}\right]
$$

where each $\rho^{X}$ is a representation of the spatial symmetry group of $(X, p)$. In particular evaluating traces of the representations of the identity symmetry gives the Calladine-Maxwell identity for $(X, p)$, while evaluating at a reflection symmetry, $g$ say, gives an identity which we write as

$$
m_{X}^{g}-s_{X}^{g}=0-b_{X}^{g}+1 .
$$

Here $b_{X}^{g}=\operatorname{trace}\left(\rho_{e}^{X}(g)\right)$ is the number of framework edges (bars) left invariant by $g$. The term 0 arises from $\operatorname{trace}\left(\rho_{s p}^{X}(g)\right)=0$, and for the three-dimensional representation $\rho_{\text {rig }}^{X}$ we have $\operatorname{trace}\left(\rho_{\text {rig }}^{X}(g)\right)=-1$.

We now exploit the evident fact that the natural inclusion $\mathcal{H}_{e}^{X} \subseteq \mathcal{H}_{e}^{G}$ respects stresses, that is, $\mathcal{H}_{s t}^{X} \subseteq \mathcal{H}_{s t}^{G}$. This is simply because a vector in the cokernel of $R(X, p)$ extends trivially to a vector in the cokernel of $R(G, p)$. The following theorem gives a family of necessary conditions all of which are computable by simple counting.

Combining these facts we obtain

Theorem 5.1. Let $(G, p)$ be a proper isostatic framework in $\mathbb{R}^{d}$. Then

(i) for each proper subframework $(X, p)$ and each spatial symmetry $g$ of $(X, p)$ we have

$$
\left|\operatorname{trace}(g) \cdot v_{X}^{g}-e_{X}^{g}-\operatorname{trace}\left(\rho_{\text {rig }}(g)\right)\right| \leq d v_{X}-e_{X}-d(d+1) / 2
$$

where $v_{X}^{g}$ (resp. $e_{X}^{g}$ ) is the number of vertices (resp. edges) in the graph $X$ that are unmoved by the symmetry.

(ii) For planar frameworks a necessary condition for isostaticity is that for each reflection symmetry $g$ of a subframework $(X, p)$

$$
\left|-e_{X}^{g}+1\right| \leq 2 v_{X}-e_{X}-3 .
$$


Proof. In $(X, p)$ we have

$$
m_{X}=d v_{X}-e_{X}-d(d+1) / 2,
$$

which follows on evaluating the general formula at the identity symmetry and noting as above that $s_{X}=0$. For the symmetry $g$ of $(X, p)$ we have $\left|m_{X}^{g}\right| \leq m_{X}$, since $m_{X}$ is the dimension of the mechanism space of $(X, p)$. On the other hand the evaluation of traces on the identity element gives

$$
m_{X}^{g}-s_{X}^{g}=\operatorname{trace}(g) \cdot v_{X}^{g}-e_{X}^{g}-\operatorname{trace}\left(\rho_{\text {rig }}(g)\right)
$$

Combining these facts we obtain (i), from which (ii) follows.

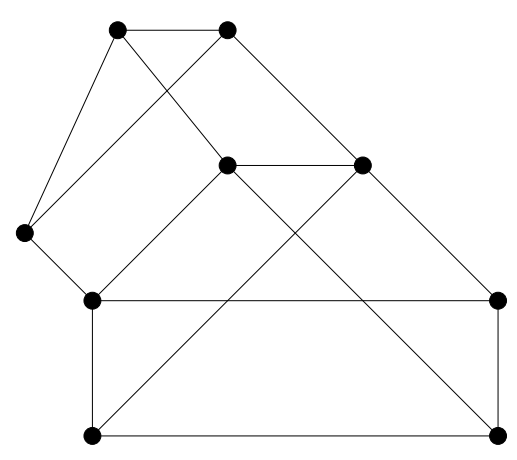

FiguRE 4. A framework with reflection symmetry in a sub-graph and a singular Jacobian.

The second part of the theorem is illustrated in Figure 4 where there is an evident subframework $X$ with six vertices with a mirror symmetry. Since the inequality of the theorem is violated for $X$ the entire framework fails to be isostatic.

5.2. Partition symmetry. We now show how symmetries associated with vertex partitioning can be significant for singularity. The idea here is that on removing the framework edges connecting vertices within each of the sets of a partition of $V$ one may be left with a set of "crossing" edges which has evident symmetry. In this event one can add edges to create complete graph frameworks within the partition sets thereby creating a body framework. If, by symmetry and counting conditions, the resulting framework has proper flexes then the original framework inherits the same proper flexes. This situation occurs for example in the simple framework of Figure 5.

More precisely let $G=(V, p)$ be a framework in $\mathbb{R}^{2}$, where each vertex has degree greater than 1 , and let $V_{1}, \ldots, V_{n}$ be a partition of $V$. Let

$$
\mathcal{S}=\left\{V_{1}, \ldots, V_{n}, e_{1}, \ldots, e_{m}\right\}
$$


where $e_{1}, \ldots, e_{m}$ are the edges of $G$ which have vertices in distinct partition sets. Delete from $p$ the framework points which are not endpoints of the edges $e_{i}$ to create a framework vector $p^{\prime}$ (representing pins). Then $\left(\mathcal{S}, p^{\prime}\right)$ is a body framework and we say that it is derived from $(G, p)$, or that it is a partition-derived body framework. Note that for a trivially derived body framework, where each partition set is a singleton, the total number of point body occurrences is the sum of the degrees of the vertices in $G$, which is $2 e$. Thus $c=2 e$ and the isostatic condition in the trivially derived framework gives $2 c=2 n+3(e-1)$, which implies $e=2 n-3$ as expected.

The following theorem, together with Theorem 4.5 give necessary conditions for isostaticity.

Theorem 5.2. Let $(G, p)$ be a framework in $\mathbb{R}^{2}$ and let $(\mathcal{S}, p)$ be a partition-derived body bar framework. Then

(i) a (non-trivial) flex of $(\mathcal{S}, p)$ gives a (non-trivial) flex of $(G, p)$.

(ii) if $(G, p)$ is isostatic then a reflection symmetry of $(\mathcal{S}, p)$ fixes exactly one edge of $(\mathcal{S}, p)$.

Proof. Let the set of velocity vectors $\left\{u_{i}, v_{e}, a_{e}\right\}$ be a flex of $(\mathcal{S}, p)$. For any two points $p_{i}$ and $p_{j}$ in body $e, u_{i}=v_{e}+a_{e}\left(p_{i}-p_{e}\right)^{\pi / 2}, u_{j}=$ $v_{e}+a_{e}\left(p_{j}-p_{e}\right)$. Thus $u_{i}-u_{j}=a_{e}(p i-p j)^{\pi / 2}$ and $\left(u_{i}-u_{j}\right) \cdot\left(p_{i}-p_{j}\right)=0$. Since every pair of points joined by a framework edge are both in some body of $\mathcal{S}$ it follows that the set $\left\{u_{i}\right\}$ is a flex of $(G, p)$. Now (i) follows and (ii) follows from (i).

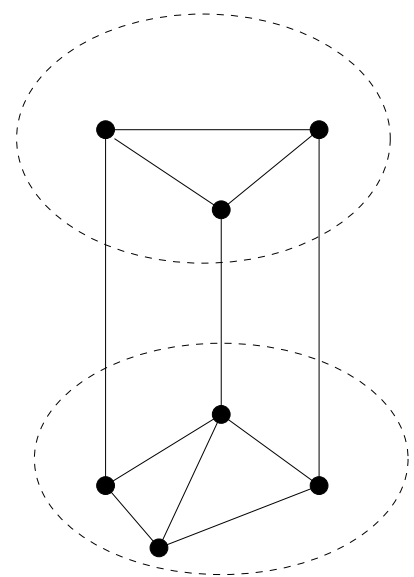

FiguRE 5. A framework with vertical reflection symmetry in a partition derived graph and a singular Jacobian. 


\section{REFERENCES}

[1] C.R. Calladine. Buckminster Fuller's Tensegrity structures and Clerk Maxwell's rules for the construction of stiff frames International Journal of Solids and Structures 14 (1978) p. 161.

[2] P.W. Fowler and S.D. Guest, "A symmetry extension of Maxwell's rule for rigidity of frames", International Journal of Solids and Structures 37 (2000) 1793-1804.

[3] A. Ceulemans and P.W. Fowler, Extension of Euler's theorem to symmetry properties of polyhedra, Nature, 353 (1991) 52 - 54.

[4] Kangwai, R.D., Guest, S.D., Symmetry-adapted equilibrium matrices, International Journal of Solids and Structures 37 (2000) 1525-1548

[5] Kangwai, R.D., Guest, S.D., Pellegrino, S., An introduction to the analysis of symmetric structures. Computers and Structures 71 (1999) 671-688.

[6] B. Schulze, Block-diagonalised rigidity matrices of symmetric frameworks and applications, preprint 2009, arXiv:0906.3377.

[7] R.Connelly, P.W.Fowler, S.D.Guest, B.Schulze, W.J.Whiteley, "When is a pinjointed framework isostatic?" International J. of Solids and Structures, 46 (2009) 762773 .

[8] G. Laman, On graphs and the rigidity of plane skeletal structures, J. Engineering Mathematics, 4 (1970) 331-340.

[9] B. Schulze, Symmetric versions of Laman's theorem, preprint, arXiv:0907.1958.

[10] L. Asimow and B. Roth, The rigidity of graphs, Trans. Amer. Math. Soc., 245 (1978), 279-289.

[11] J. Graver, B. Servatius and H. Servatius, Combinatorial rigidity, Graduate Texts in Mathematics, vol 2, Amer. Math. Soc., 1993.

[12] T.S. Tay and W. Whitely, Recent advances in the generic rigidity of structures, Structural Topology 9, 1984, 31-38.

[13] B. Jackson and T. Jordan, The generic rank of body-bar-and-hinge frameworks, European J. Combinatorics 31 (2010) 574-588.

[14] J.C. Owen, Algebraic solution for geometry from dimensional constraints, in ACM Symposium on Foundations in Solid Modeling, pages 397-407, Austen, Texas, 1991.

[15] J.C. Owen and S.C. Power, The non-solvability by radicals of generic 3connected planar Laman graphs, Trans. Amer. Math. Soc., 359 (2007) 22692303.

[16] W. Whiteley, Rigidity and scene analysis, Handbook of Discrete and Computational Geometry, eds J.E. Goodman and J. O'Rourke, CRC Press, 1997.

[17] J.C. Owen and S.C. Power, Infinite Bar-Joint Frameworks, Proceedings of the Symposium in Applied Computing, SAC2009 (Honolulu, March 2009).

[18] J.C. Owen and S.C. Power, Continuous curves from infinite Kempe linkages, Bull. LMS 2009 ; doi: 10.1112/blms/bdp087.

[19] J.C. Owen and S.C. Power, Infinite bar-joint frameworks, crystals and operator theory, preprint 2010.

[20] A. Donev and S. Torquato, Energy-efficient actuation in infinite lattice structures, J. Mech Phys. Solids, 51 (2003) 1459-1475.

[21] S. D. Guest and J. W. Hutchinson, On the determinacy of repetitive structures, Journal of the Mechanics and Physics of Solids 51 (2003) 383391.

[22] A. Ceulemans, L. F. Chibotaru, P. W. Fowler and M. Szopa, Symmetry extensions of Eulers polyhedral theorem and the band theory of solids, Journal of Chemical Physics, 14 (1999) 6916-6926.

[23] C.S. Borcea and I. Streinu, Periodic frameworks and flexibility, Proc. R. Soc. A, doi:10.1098/rspa.2009.0676. 
[24] E. Ross, private communication.

D-Cubed, Siemens PlM Software, Park House, Castle Park, CamBRIDGE, UNITED Kindom, OWEN.JOHN.EXT@SIEMENS.COM

Department of Mathematics and Statistics, LAncaster University,, LANCASTER, LA1 4YF, United Kingdom, s.POWER@LANCASTER.AC.UK 